\title{
ON LACUNARY TRIGONOMETRIC SERIES
}

BY

\section{ANTONI ZYGMUND*}

1. Fundamental theorem. In a recent paper $\dagger I$ have proved the theorem that if a lacunary trigonometric series

$$
\sum_{k=1}^{\infty}\left(a_{k} \cos n_{k} \theta+b_{k} \sin n_{k} \theta\right) \quad\left(n_{k+1} / n_{k}>q>1,0 \leqq \theta \leqq 2 \pi\right)
$$

has its partial sums uniformly bounded on a set of $\theta$ of positive measure, then the series

$$
\sum_{k=1}^{\infty}\left(a_{k}^{2}+b_{k}^{2}\right)
$$

converges. The proof was based on the following lemma (which was not stated explicitly but is contained in the paper referred to, pp. 91-94).

LEMMA 1. Let $E$ be an arbitrary measurable set of points of the interval $(0,2 \pi), m(E)>0$. Then there exists a number $N_{0}=N_{0}(q, E)$ such that, for $N>N_{0}$, we have

$$
\int_{E}\left|s_{N}-s_{N_{0}}\right|^{2} d \theta \geqq \frac{1}{4} m(E) \sum_{k=N_{0}+1}^{N}\left(a_{k}^{2}+b_{k}^{2}\right),
$$

where $s_{N}$ denotes the Nth partial sum of the series (1), i.e.

$$
s_{N}=\sum_{k=1}^{N}\left(a_{k} \cos n_{k} \theta+b_{k} \sin n_{k} \theta\right) .
$$

Now we shall prove a somewhat more general theorem.

TheOREM 1. If the partial sums of the series (1) are uniformly bounded below on a set $E$ of positive measure, then series (2) converges.

If $A$ is a positive constant, sufficiently large, we have $s_{N}+A \geqq 0$ on $E$, and so

$$
\begin{aligned}
\int_{E}\left|s_{N}\right| d \theta & \leqq \int_{E}\left(\left|s_{N}+A\right|+A\right) d \theta=2 A m(E)+\int_{E} s_{N} d \theta \\
& =2 A m(E)+\sum_{k=1}^{N}\left(a_{k} \xi_{k}+b_{k} \eta_{k}\right),
\end{aligned}
$$

* Presented to the Society, March 25, 1932; received by the editors January 4, 1932; $\$ \$ 4$ and 5 received February $11,1932$.

† On the convergence of lacunary trigonometric series, Fundamenta Mathematicae, vol. 16 (1930), pp. 90-107. 
where

$$
\xi_{m}=\int_{E} \cos m \theta d \theta, \quad \eta_{m}=\int_{E} \sin m \theta d \theta .
$$

By Schwarz's inequality the absolute value of the last sum in (4) does not exceed

$$
\begin{aligned}
\sum_{k=1}^{N}\left(a_{k}^{2}+b_{k}^{2}\right)^{1 / 2}\left(\xi_{n_{k}}^{2}+\eta_{n_{k}}^{2}\right)^{1 / 2} & \leqq\left\{\sum_{k=1}^{N}\left(a_{k}^{2}+b_{k}^{2}\right)\right\}^{1 / 2}\left\{\sum_{k=1}^{N}\left(\xi_{n_{k}}^{2}+\eta_{n_{k}}^{2}\right)\right\}^{1 / 2} \\
& =\mu_{N}\left\{\sum_{k=1}^{N}\left(\xi_{n_{k}}^{2}+\eta_{n_{k}}^{2}\right)\right\}^{1 / 2}
\end{aligned}
$$

where $\mu_{N}{ }^{2}$ denotes the $N$ th partial sum of the series (2). The series

$$
\sum\left(\xi_{m}^{2}+\eta_{m}^{2}\right)
$$

being convergent, it is not difficult to see that, if we suppose that $\mu_{N} \rightarrow \infty$, then

$$
\int_{E}\left|s_{N}\right| d \theta=o\left(\mu_{N}\right)
$$

It is familiar that, if $f$ is (say) bounded, then the logarithm of the integral of $|f|^{\alpha}$ (extended over a set $E$ ) is a convex function of $\alpha$. Consequently, we have

$$
\int_{E}\left|s_{N}\right|^{2} d \theta \leqq\left(\int_{E}\left|s_{N}\right| d \theta\right)^{2 / 3}\left(\int_{E}\left|s_{N}\right|^{4} d \theta\right)^{1 / 3} .
$$

But, if we use $C$ as a generic notation for a positive constant independent of $N$, we have by Lemma 1 for $N$ sufficiently large,

$$
\int_{E}\left|s_{N}\right|^{2} d \theta \geqq C \mu_{N}^{2}
$$

On the other hand, it is known $\uparrow$ that

Hence

$$
\int_{E}\left|s_{N}\right|^{4} d \theta \leqq C \mu_{N}{ }^{4}
$$

$$
\int_{E}\left|s_{N}\right| d \theta \geqq C \mu_{N},
$$

* See, e.g., Hausdorff, Mathematische Zeitschrift, vol. 16 (1923), p. 165.

$\dagger$ Zygmund, Fundamenta Mathematicae, loc. cit., Theorem F. See also R.E.A.C. Paley, Proceedings of the London Mathematical Society, vol. 31 (1930), pp. 301-328, Theorem 4. 
contradicting (5). Consequently $\mu_{N}=O(1)$; and the theorem is proved.

Let

$$
s_{N}^{+}=\max \left(s_{N}, 0\right), s_{N^{-}}=\min \left(s_{N}, 0\right) .
$$

The following proposition is stated for the sake of completeness and is not used in the sequel.

LEMмa 2. If $\mu_{N} \rightarrow \infty$, then the relations

$$
s_{N}{ }^{+}=o\left(\mu_{N}\right), s_{N}^{-}=o\left(\mu_{N}\right)
$$

may be true only on a set of measure zero.

Otherwise, there would exist a set $E$ of points of positive measure and a sequence of numbers $\eta_{1}, \eta_{2}, \cdots, \eta_{N}, \cdots$ tending to zero, such that we have, say,

$$
\left|s_{N}-\right| \leqq \eta_{N} \mu_{N}, \quad \theta \subset E .
$$

Putting $A=\eta_{N} \mu_{N}$ we get again the inequality (5) contradictory to (6).

Theorem 1 may be stated also in the following completely equivalent form:

TheOREm $1^{\prime}$. If the series (2) diverges, then, for almost all $\theta$, we have simultaneously

$$
\lim _{N \rightarrow \infty} s_{N}=-\infty, \lim _{N \rightarrow \infty} s_{N}=+\infty .
$$

Theorem 1 remains true if, instead of ordinary partial sums, we consider Toeplitz's sums. We only state the theorem since the proof is not essentially different from that of Theorem 1 and may be left to the reader.

TheOREM 2. If the Toeplitz $\left(T^{*}\right) \dagger$ sums $\sigma_{N}$ of the series (1) are uniformly bounded below on a set $E$ of positive measure, the series (2) converges.

The condition of $\sigma_{N}$ being bounded below may be replaced by a less stringent one, analogous to (7).

We might observe finally that, if the series (2) converges, and $t_{N}$ and $\rho_{N}{ }^{2}$ are remainders of (1) and (2), then the relations $t_{N}{ }^{+}=o\left(\rho_{N}\right), t_{N}{ }^{-}=o\left(\rho_{N}\right)$ are false almost everywhere.

2. Infinite trigonometric products. Using Theorem 1 we can prove some theorems concerning the infinite products $\ddagger$

$$
\prod_{k=1}^{\infty}\left(1+a_{k} \cos n_{k} x\right)
$$

$\dagger$ Zygmund, Fundamenta Mathematicae, loc. cit., p. 96.

$\ddagger$ Considered for the first time by F. Riesz, Mathematische Zeitschrift, vol. 2 (1918), pp. 312-315. 
where the integers $n_{k}$ satisfy the condition

$$
n_{k+1} / n_{k}>q>3 \text {, }
$$

and the constants $a_{k}$ do not exceed 1 in absolute value. Only for the sake of simplicity we do not replace in (9) $\cos n_{k} x$ by $\cos \left(n_{k} x+\xi_{k}\right)$, where $\xi_{1}$, $\xi_{2}, \cdots$ are arbitrary real constants. For such more general products the arguments would be exactly the same.

Multiplying formally, and taking into account that no two or more terms cancel each other, we represent the product (9) in the form of a trigonometric series

$$
1+\sum_{\nu=1}^{\infty} c_{\nu} \cos \nu x,
$$

where $c_{\nu}=0$ if $\nu$ is not of the form $n_{k}+\epsilon_{1} n_{k-1}+\cdots+\epsilon_{k-1} n_{1}$ with $\epsilon_{i}=0,-1$, +1 . Let

$$
\mu_{k}^{\prime}=n_{k+1}-n_{k}-\cdots-n_{1}, \mu_{k}=n_{k}+n_{k-1}+\cdots+n_{1} .
$$

Since $n_{k+1} / n_{k}>q=3+\epsilon$ we see that $\mu_{k+1}^{\prime} / \mu_{k}>1+\epsilon$. Thus, the series (10), although not lacunary, contains infinitely many gaps. If $\sum a_{k}^{2}<\infty$ then $\sum c_{\nu}^{2}<\infty$ and the series (10) belongs to $L^{2}$. In what follows we shall always suppose that $\sum a_{k}^{2}=\infty$. Denoting by

$$
s_{N}=1+\sum_{\nu=1}^{N} c_{\nu} \cos \nu \theta
$$

the $N$ th partial sum of the series (10) and by $p_{N}$ the $N$ th partial product of (9), we see that $s_{\mu_{N}}=p_{N}$. It is well known that if, for a trigonometric series with the partial sums $s_{\nu}$, the sequence

$$
\int_{0}^{2 \pi}\left|s_{\nu}\right| d \theta
$$

or even a subsequence of (11), is bounded, the series, without its constant term, is the differentiated Fourier series of a function $G$ of bounded variation, and, in particular is summable $(C, 1)$ almost everywhere. In our case

$$
\int_{0}^{2 \pi}\left|s_{\mu_{N}}\right| d \theta=\int_{0}^{2 \pi} p_{N} d \theta=\int_{0}^{2 \pi}(1+\cdots) d \theta=2 \pi .
$$

It is easy to see that $\sum\left|c_{\nu}\right| / \nu$ is convergent since the group of terms of this series with $\nu=n_{k}+\epsilon_{1} n_{k-1}+\cdots+\epsilon_{k-1} n_{1}\left(\epsilon_{i}=0, \pm 1\right)$ is $O\left(2^{k} / n_{k}\right)$. Hence the function $G(x)$ is everywhere continuous. Let us put $F(x)=G(x)+x(0 \leqq x$ $\leqq 2 \pi)$. Then 


$$
1=\frac{1}{2 \pi} \int_{0}^{2 \pi} d F, \quad c_{\nu}=\frac{1}{\pi} \int_{0}^{2 \pi} \cos \nu x d F,
$$

i.e. the series (10) is the Fourier-Stieltjes series of $F$. As $p_{N} \geqq 0$, and

$$
F(x)-F(0)=\lim _{N_{k} \rightarrow \infty} \int_{0}^{x} p_{N_{k}}(\theta) d \theta,
$$

we see that $F(x)$ is an increasing function.

In view of Lemma 3 below,

$$
p(\theta)=\lim _{N \rightarrow \infty} p_{N}(\theta)=\lim _{N \rightarrow \infty} s_{\mu_{N}}(\theta)
$$

exists almost everywhere. We shall prove that $p(\theta)=0$, almost everywhere.

In fact, if

$$
\varlimsup_{k \rightarrow \infty} a_{k}>0 \quad \text { or } \quad \varliminf_{k \rightarrow \infty} a_{k}<0
$$

and if $p\left(\theta_{0}\right) \neq 0$, then

$$
1+a_{k} \cos n_{k} \theta_{0}=p_{k}\left(\theta_{0}\right) / p_{k-1}\left(\theta_{0}\right) \rightarrow 1 \text {. }
$$

Consequently $a_{k} \cos n_{k} \theta_{0} \rightarrow 0$ which may be true only on a set of $\theta_{0}$ of measure 0 . If $a_{k} \rightarrow 0$, then, except at a finite number of points, we have

$$
\begin{aligned}
p_{N} & =\exp \left[\sum_{k=1}^{N} \log \left(1+a_{k} \cos n_{k} \theta\right)\right] \\
& =\exp \left[\sum_{k=1}^{N} a_{k} \cos n_{k} \theta-\frac{1}{2} \sum_{k=1}^{N} a_{k^{2}} \cos ^{2} n_{k} \theta+o\left(\sum_{k=1}^{N}\left|a_{k}\right|^{3}\right)\right] \\
& =\exp \left[\sum_{k=1}^{N} a_{k} \cos n_{k} \theta-\frac{1}{4} \sum_{k=1}^{N} a_{k}^{2} \cos 2 n_{k} \theta-\frac{1}{4} \sum_{k=1}^{N} a_{k}^{2}+O\left(\sum_{k=1}^{N}\left|a_{k}\right|^{3}\right)\right] .
\end{aligned}
$$

The sum of the series

$$
\sum_{k=1}^{\infty} a_{k} \cos n_{k} \theta, \quad-\frac{1}{4} \sum_{k=1}^{\infty} a_{k}^{2} \cos 2 n_{k} \theta
$$

is itself a lacunary series

$$
\sum_{k=1}^{\infty} a_{k}^{\prime} \cos \lambda_{k} \theta
$$

and, as 


$$
\sum_{1}^{N}\left|a_{k}\right|^{3}=o\left(\sum_{1}^{N}\left|a_{k}\right|^{2}\right)
$$

we may write the expression (12) in the form

$$
\exp \left[\sum_{k=1}^{2 N} a_{k}^{\prime} \cos \lambda_{k} \theta-\frac{1}{4}\left(1+\delta_{N}\right) \sum_{k=1}^{N} a_{k}^{2}\right],
$$

where $\delta_{N} \rightarrow 0$. As $\sum a_{k}{ }^{2}=\infty$, the partial sums of the series (13) are, by Theorem 1 , unbounded below for almost all $\theta$, and so, as the limit of the expression (14) exists almost everywhere, it must be equal to zero for almost all $\theta$.

Thus we have obtained the following theorem.

THEOREM 3. If

$$
\left|a_{k}\right| \leqq 1, n_{k+1} / n_{k}>q>3, \sum a_{k}^{2}=\infty,
$$

the infinite product (9), written in the form of the trigonometric series (10), is the Fourier-Stieltjes series of a function $F(x)$, continuous, monotonically increasing, with almost everywhere vanishing derivative $F^{\prime}$. The partial sums $s_{\mu_{k}}, \mu_{k}=n_{k}$ $+\cdots+n_{1}$, of the series (10) converge almost everywhere to 0 .

The conclusion above, that $\lim p_{N}(\theta)=\lim s_{\mu_{N}}(\theta)$ exists almost everywhere, is easily derived from the following lemma which is well known, although it is difficult to make any reference.

Lemma 3. If the series $\sum_{0}^{\infty} \alpha_{\nu}$ is summable $(C, 1)$ to a sum $s$, and possesses infinitely many gaps $\left(\nu_{k}, \nu_{k}^{\prime}\right)$ with $\nu_{k}^{\prime} / \nu_{k}>1+\epsilon$, then the sequence of partial sums $s_{\nu_{k}}$ of the series converges to the same limit s.

Let

$$
\begin{gathered}
s_{n}=\alpha_{0}+\alpha_{1}+\cdots+\alpha_{n}, s_{n}=s_{\nu_{k^{\prime}}} \text { when } \nu_{k} \leqq n \leqq \nu_{k}^{\prime}, \\
\sigma_{n}=\left(s_{0}+s_{1}+\cdots+s_{n}\right) /(n+1),
\end{gathered}
$$

and assume for simplicity, without any loss of generality, that

$$
s=\lim _{n \rightarrow \infty} \sigma_{n}=0 .
$$

Then

$$
s_{\nu_{k^{\prime}}}\left(\nu_{k}^{\prime}-\nu_{k}+1\right)=\left(\nu_{k}^{\prime}+1\right) \sigma_{\nu_{k^{\prime}}}-\nu_{k} \sigma_{\nu_{k-1}-1}=o\left(\nu_{k}^{\prime}\right)
$$

whence

$$
\lim s_{\nu_{k^{\prime}}}=0 .
$$

3. A case of convergence of series (10). When $a_{k} \rightarrow 0$ we can prove more than has been stated in Theorem 3 . 
THEOREM 4. If

$$
\lim a_{k}=0, n_{k+1} / n_{k}>q>3, \sum a_{k}^{2}=\infty,
$$

the trigonometric series (10) obtained from the product (9) converges to zero almost everywhere.

Thus we get a simple example of a trigonometric series converging almost everywhere, but of course not everywhere, to zero. The first example of such series was constructed (by a quite different method) by Menchoff.* We assume for simplicity that $\left|a_{k}\right| \leqq 1, k=1,2, \cdots$, and prove

LEмма 4. Not only the partial sums $s_{\mu_{k}}, \mu_{k}=n_{k}+\cdots+n_{1}$, but also the partial sums $\tilde{s}_{\mu_{k}}$ of the series conjugate to (10) converge almost everywhere.

For the sake of completeness we give the proof, although the result is contained in a paper by R.E.A.C. Paley and myself. $\dagger$ Let $s_{n}, \sigma_{n}, \tilde{s}_{n}, \tilde{\sigma}_{n}$ denote respectively the $n$th partial sum and the $n$th Cesàro means of the series (10) and of its conjugate.

Denoting by $K_{n}$ Fejér's kernel, we have $\ddagger$

$$
\begin{aligned}
\bar{s}_{n}(\theta)-\tilde{\sigma}_{n}(\theta) & =-s_{n}^{\prime}(\theta) /(n+1) \\
& =-\frac{1}{\pi(n+1)} \int_{0}^{2 \pi} s_{n}(\theta+u)[\sin u+2 \sin 2 u+\cdots+n \sin n u] d u \\
& =-\frac{2}{\pi} \int_{0}^{2 \pi} s_{n}(\theta+u) \sin (n+1) u K_{n}(u) d u .
\end{aligned}
$$

This is readily shown by writing $\sin (n+1) u K_{n}(u)$ in the form of a trigonometric polynomial and rejecting the terms of order exceeding $n$. If $s_{n} \geqq 0$, the last expression does not exceed in absolute value

$$
\frac{2}{\pi} \int_{0}^{2 \pi} s_{n}(\theta+u) K_{n}(u) d u=2 \sigma_{n}(\theta) .
$$

In the case of the series (10) we have $s_{\mu_{k}} \geqq 0, \sigma_{n} \rightarrow 0$. Consequently the convergence of $\tilde{\sigma}_{\mu_{k}}$ implies that of $\tilde{s}_{\mu_{k}}$.

It is known, however, that $\tilde{\sigma}_{n}$ converges almost everywhere, since the conjugate of a Fourier-Stieltjes series is $(C, 1)$ summable almost everywhere. $\S$ Hence $\tilde{\sigma}_{\mu_{k}}$, and therefore $\tilde{s}_{\mu_{k}}$, converges almost everywhere.

* Comptes Rendus, vol. 163 (1916), p. 433. See also Nina Bary, Fundamenta Mathematicae, vol. 9 (1927), pp. 62-115, and A. Rajchman, Mathematische Annalen, vol. 101 (1929), pp. 686-700.

† Studia Mathematica, vol. 2 (1930), pp. 221-227.

$\ddagger$ The formula is due to F. Riesz, Comptes Rendus, vol. 158 (1914), pp. 1657-1661.

$\S$ The summability $(C, 1)$ (almost everywhere) of the series conjugate to Fourier-Stieltjes series has been proved by A. Plessner, Zur Theorie der konjugierten trigonometrischen Reihen, Mitteilungen des Mathematischen Seminars der Universität Giessen, vol. 10 (1923), pp. 1-36. 
We pass on now to the proof of Theorem 4. Let $M$ be the greater of the two numbers l.u.b. $\left|s_{\mu_{k}}\right|$ and l.u.b. $\left|\tilde{s}_{\mu_{k}}\right| ; M=M(\theta)$ is finite almost everywhere. Consider an arbitrary point $\theta$ where $M$ is finite and let $A \equiv A(k, \theta)$ be a number satisfying the inequalities

$$
\begin{aligned}
\left(15_{k-1}\right) \quad & \left|\sum_{\nu=\lambda}^{\mu_{k-1}} c_{\nu} \cos \nu \theta\right| \leqq A, \quad\left|\sum_{\nu=\lambda}^{\mu_{k-1}} c_{\nu} \sin \nu \theta\right| \leqq A \quad\left(1 \leqq \lambda \leqq \mu_{k-1}\right), \\
\left(16_{k-1}\right) \quad \mid & \left|\sum_{\nu=\lambda}^{\mu_{i}} c_{\nu} \cos \nu \theta\right| \leqq A-2 M, \quad\left|\sum_{\nu=\lambda}^{\mu_{i}} c_{\nu} \sin \nu \theta\right| \leqq A-2 M \\
& \left(\mu_{i-1}<\lambda \leqq \mu_{i}, i=1,2, \cdots, k-1 ; \mu_{0}=0\right) .
\end{aligned}
$$

As regards the partial sums of the series (10) it is obvious that

$$
\begin{aligned}
s_{\mu_{k}} & =\left(1+\sum_{\nu=1}^{\mu_{k-1}} c_{\nu} \cos \nu \theta\right)\left(1+a_{k} \cos n_{k} \theta\right) \\
& =s_{\mu_{k-1}}+a_{k} \cos n_{k} \theta+\frac{1}{2} a_{k} \sum_{\nu=1}^{\mu_{k-1}} c_{\nu}\left[\cos \left(n_{k}-\nu\right) \theta+\cos \left(n_{k}+\nu\right) \theta\right] .
\end{aligned}
$$

Let us consider separately the following two cases: (1) $\mu_{k-1}<\lambda<n_{k}$; (2) $n_{k} \leqq \lambda \leqq \mu_{k}$. In the first case

$$
s_{\lambda}=s_{\mu_{k-1}}+\frac{1}{2} a_{k} \sum_{\nu=n_{k}-\lambda}^{\mu_{k-1}} c_{\nu} \cos \left(n_{k}-\nu\right) \theta, s_{\lambda}=s_{\mu_{k-1}},
$$

according as $\lambda \geqq n_{k}-\mu_{k-1}$ or $\lambda<n_{k}-\mu_{k-1}$. The last sum, being equal to

$$
\frac{1}{2} a_{k} \Re\left[e^{-i n_{k} \theta} \sum_{\nu=n_{k}-\lambda}^{\mu_{k-1}} c_{\nu} e^{i \nu \theta}\right]
$$

does not exceed in absolute value

$$
\frac{1}{2}\left|a_{k}\right|\left|\sum_{\nu=n_{k}-\lambda}^{\mu_{k-1}} c_{\nu} e^{i \nu \theta}\right| \leqq \frac{1}{2}\left|a_{k}\right| A \cdot 2^{1 / 2} .
$$

In the second case we have

$$
\begin{aligned}
s_{\lambda} & =s_{\mu_{k}}-\frac{1}{2} a_{k} \sum_{\nu=\lambda-n_{k}}^{\mu_{k-1}} c_{\nu} \cos \left(n_{k}+\nu\right) \theta, \quad \text { if } \lambda>n_{k}, \\
s_{n_{k}} & =s_{n_{k}+1}-a_{k} \cos n_{k} \theta .
\end{aligned}
$$

Argueing as in the first case, we see that now $\left|s_{\lambda}-s_{\mu_{k}}\right|$ does not exceed

$$
\left|a_{k}\right|+\frac{1}{2}\left|a_{k}\right| A \cdot 2^{1 / 2} \text {. }
$$


Consequently, for any $\lambda$ on the range $\left(\mu_{k-1}+1, \mu_{k}\right)$ we have one of the inequalities

$$
\left|s_{\lambda}\right| \leqq\left\{\begin{array}{l}
\left|s_{\mu_{k-1}}\right|+\left|a_{k}\right| A / 2^{1 / 2} \\
\left|s_{\mu_{k}}\right|+\left|a_{k}\right|\left(1+A / 2^{1 / 2}\right) \leqq M+\left|a_{k}\right|\left(1+A / 2^{1 / 2}\right) .
\end{array}\right.
$$

The same inequalities hold for the conjugate partial sums, which we obtain by replacing in (17) and (18) cosines by sines. We may, of course, take $A$ arbitrarily large. In particular we may take $A$ so large that

$$
2 M+\left(1+A / 2^{1 / 2}\right) \leqq A-2 M .
$$

Then, from (19) we see that, if $\mu_{k-1}<\lambda \leqq \mu_{k}$, we have

$$
\left|\sum_{\nu=\lambda}^{\mu_{k}} c_{\nu} \cos \nu \theta\right| \leqq\left|s_{\mu_{k}}\right|+\left|s_{\lambda-1}\right| \leqq M+M+\left(1+A / 2^{1 / 2}\right) \leqq A-2 M ;
$$

consequently, as the argument with the conjugate series is exactly the same, the inequalities $\left(15_{k-1}\right)$ and $\left(16_{k-1}\right)$ involve. $\left(16_{k}\right)$. If $\mu_{j-1}<\lambda \leqq \mu_{j}, j<k$, then

$$
\left|\sum_{\nu=\lambda}^{\mu_{k}} c_{\nu} \cos \nu \theta\right| \leqq\left|\sum_{\lambda}^{\mu_{j}}\right|+\left|\sum_{\mu_{j}+1}^{\mu_{k}}\right| \leqq A-2 M+2 M=A .
$$

Hence $\left(15_{k}\right)$ and $\left(16_{k}\right)$ follow from $\left(15_{k-1}\right)$ and $\left(16_{k-1}\right)$, which shows that they are true for every value of $k$. In particular (even if $a_{k}$ does not tend to zero) $s_{\lambda}$ and $\tilde{s}_{\lambda}$ are bounded. If $a_{k} \rightarrow 0$ the sequences $\left\{s_{\lambda}\right\}$ and $\left\{\tilde{s}_{\lambda}\right\}$ are convergent, as follows, e.g. for the former, from the inequalities (19).

4. Approximately differentiable lacunary series. In my paper referred to, I have proved that, if a lacunary trigonometric series (1) is the Fourier series of a function $F(\theta)$ differentiable on a set $E$ of points of positive measure, or even if only

$$
\lim _{h \rightarrow 0} \frac{F(\theta+h)-F(\theta-h)}{2 h}
$$

exists, and is finite on $E$, then the series

$$
\sum\left(a_{k}^{2}+b_{k}^{2}\right) n_{k}^{2}
$$

converges, that is, $F(\theta)$ is the integral of a function $f(\theta)$ of the class $L^{2}$.* The theorem and the proof hold if in the expression (20) the variable $h$ tends to zero through an arbitrary, but fixed, sequence of positive values, $h_{1}$, $h_{2}, \cdots, h_{v}, \cdots$. Hence, if the series (1) converges absolutely and the series (21) diverges, the ratio

* Zygmund, Fundamenta Mathematicae, loc. cit., Theorem C, p. 95. 


$$
\frac{F\left(\theta+h_{i}\right)-F\left(\theta-h_{i}\right)}{2 h_{i}}
$$

does not tend to any finite limit for almost every $\theta$. Essentially the same proof shows that the expressions

$$
\frac{F\left(\theta+h_{i}\right)-F(\theta)}{h_{i}}, \frac{F(\theta)-F\left(\theta-h_{i}\right)}{h_{i}}
$$

may have a limit only in a set of points of measure zero.* It may be asked if the theorem remains true, supposing that $F(\theta)$ possesses on a set $E$ of positive measure an approximate derivative $F^{[1]}(\theta)$, defined as

$$
\lim \frac{F(\theta+h)-F(\theta)}{h}
$$

where $h$ tends to \pm 0 remaining on a set $H \equiv H(\theta)$ having 0 as its point of density. We have in fact the following

TheOREM 5. If series (1) is the Fourier series of a function $F(\theta)$ approximately differentiable on a set $E$ of positive measure, the series (21) converges.

In view of the preceding remarks the proof follows immediately from the following lemma of Khintchine:

LEMMA 5. If a function $F(\theta), 0 \leqq \theta \leqq 2 \pi$, is approximately differentiable on a set $E$ of positive measure, there exists a sequence of positive numbers $h_{1}, h_{2}, \ldots$, $h_{i}, \cdots$ such that almost everywhere on $E$ both expressions (23), and, consequently, the ratio (22) converge to $F^{[1]}(\theta) . \dagger$

In the special case where

$$
\left(a_{k}^{2}+b_{k}^{2}\right)^{1 / 2}=O\left(1 / n_{k}\right)
$$

(e.g. if $n_{k}=2^{k}, a_{k}=2^{-k}, b_{k}=0$ ) Theorem 5 may be proved without using Khintchine's lemma. In fact, it is not difficult to prove that, under condition (24), the difference

$$
\frac{F(\theta+h)-F(\theta-h)}{2 h}-\sum_{0<n_{k} \leqq 1 / h}\left(b_{k} \cos n_{k} \theta-a_{k} \sin n_{k} \theta\right) n_{k}
$$

is uniformly bounded when $h \rightarrow 0$. $\ddagger$ Let $\theta_{0}$ be an arbitrary point of $E$. Since $H\left(\theta_{0}\right)$ has 0 as a point of density, any interval $(h, q h)$ contains at least one

* The first example of continuous functions possessing the above property was given by A. Khintchine, Fundamenta Mathematicae, vol. 9 (1927), pp. 212-279, especially p. 266.

$\dagger$ Khintchine, loc. cit., pp. 259 and 269.

$\ddagger$ Zygmund, Fundamenta Mathematicae, loc. cit., p. 102. 
point of $H\left(\theta_{0}\right)$ provided that $h$ is sufficiently small. At the same time in the interval $(1 /(q h), 1 / h)$ we find at most one integer $n_{k}$. Consequently, the partial sums of the series

$$
\sum_{k=1}^{\infty}\left(b_{k} \cos n_{k} \theta-a_{k} \sin n_{k} \theta\right) n_{k}
$$

are bounded for $\theta=\theta_{0}$. As $E$ is of positive measure, the convergence of series (21) follows.

From Theorem 5 we deduce, in particular, that the well known Weierstrass non-differentiable function may have an approximate derivative only on a set of measure zero.

5. Uniqueness theorem for lacunary series. The following Theorem is proved.

THEOREM 6. If series (1) converges to zero on a set $E$ of positive measure, then the sum of this series vanishes identically.

It follows from the hypothesis, that the series (1) is the Fourier series of a function $F(\theta)$ possessing a vanishing approximate derivative almost everywhere on $E$. Consequently, $F(\theta)$ is the integral of a function $f(\theta)$, whose Fourier series is the series (25). Since $f(\theta)$ vanishes almost everywhere on $E$, the series $(25)$ is summable $(C, 1)$, and even, being a lacunary series, converges to zero almost everywhere on $E$ (Lemma 3 ). Repeating the same argument we find that $F(\theta)$ is differentiable infinitely many times, and that all its derivatives vanish everywhere on a subset $D$ of $E$, of the same measure. In particular, we have

$$
\sum_{k=1}^{\infty} n_{k}^{2 \nu}\left(a_{k} \cos n_{k} \theta+b_{k} \sin n_{k} \theta\right)=0 \quad(\theta \subset D ; \nu=1,2, \cdots) .
$$

From Lemma 1 we see that there exists a number $k_{0}$, independent of $\nu$, such that for $\nu=1,2, \cdots$,

$$
\int_{D}\left\{\sum_{k=k_{0}+1}^{\infty}\left(a_{k} \cos n_{k} \theta+b_{k} \sin n_{k} \theta\right) n_{k}{ }^{2 \nu}\right\}^{2} d \theta \geqq \frac{1}{4} m(D) \sum_{k=k_{0}+1}^{\infty} n_{k}{ }^{4 \nu}\left(a_{k}{ }^{2}+b_{k}{ }^{2}\right) .
$$

By (26) the left hand member of (27) reduces to

$$
\int_{D}\left\{\sum_{k=1}^{k_{0}}\left(a_{k} \cos n_{k} \theta+b_{k} \sin n_{k} \theta\right) n_{k}{ }^{2 \nu}\right\}^{2} d \theta=O\left(n_{k_{0}}^{4 \nu}\right) .
$$

Hence (27) is possible if and only if $a_{k}=b_{k}=0$, for $k>k_{0}$. This will hold then for all $k>0$. 
From Theorem 6 we deduce that if two integrable functions $f_{1}(\theta)$ and $f_{2}(\theta)$ are equal on a set of positive measure, and if the Fourier coefficients of $f_{1}$ and $f_{2}$ coincide, except perhaps for those of ranks $n_{1}, n_{2}, \cdots ; n_{k-1} / n_{k} \geqq q>1$, then $f_{1}(\theta)=f_{2}(\theta)$ almost everywhere.

Theorem 6 shows also that the sum of series (1) cannot have the same value on a set of positive measure, i.e. any value which is assumed by the sum of such a series, is assumed only on a set of points of measure zero. On the other hand, it has been shown that if

$$
\sum\left(\left|a_{k}\right|+\left|b_{k}\right|\right)=\infty \text { but } a_{k} \rightarrow 0, b_{k} \rightarrow 0,
$$

and if $s$ is an arbitrary real number, the series (1) converges to $s$ on a set of points which is everywhere dense. ${ }^{*}$ This is striking because it is known that series (1) diverges almost everywhere when $\sum\left(a_{k}^{2}+b_{k}^{2}\right)=\infty$.

* Zygmund, Studia Mathematica, vol. 3 (1931), pp. 77-91, especially p. 82.

UNIVERSITY OF VILNA,

Vilna, Poland 